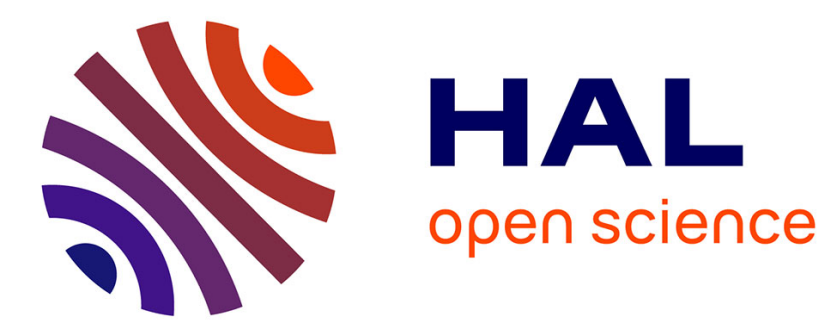

\title{
Impact du cancer ORL sur la sociabilité des conjoints
}

\author{
G. Grandazzi, L.-M. Roussel, F. Cuny, F. Morlais, L. Launay, E. Babin
}

\section{To cite this version:}

G. Grandazzi, L.-M. Roussel, F. Cuny, F. Morlais, L. Launay, et al.. Impact du cancer ORL sur la sociabilité des conjoints. Annales françaises d'Oto-rhino-laryngologie et de Pathologie Cervico-faciale, 2019, 136, pp.164 - 168. 10.1016/j.aforl.2018.05.009 . hal-03484412

\section{HAL Id: hal-03484412 \\ https://hal.science/hal-03484412}

Submitted on 20 Dec 2021

HAL is a multi-disciplinary open access archive for the deposit and dissemination of scientific research documents, whether they are published or not. The documents may come from teaching and research institutions in France or abroad, or from public or private research centers.
L'archive ouverte pluridisciplinaire $\mathbf{H A L}$, est destinée au dépôt et à la diffusion de documents scientifiques de niveau recherche, publiés ou non, émanant des établissements d'enseignement et de recherche français ou étrangers, des laboratoires publics ou privés.

\section{다)(1) $(5$}

Distributed under a Creative Commons Attribution - NonCommercial| 4.0 International 


\title{
Impact du cancer ORL sur la sociabilité des conjoints
}

Guillaume Grandazzi ${ }^{1}$, Lise-Marie Roussel ${ }^{1,2^{*}}$, Florence Cuny ${ }^{2}$, Fabrice Morlais ${ }^{1}$, Ludivine Launay ${ }^{1}$, Emmanuel Babin ${ }^{1,2}$

${ }^{1}$ INSERM / UNICAEN U1086, Cancers et Préventions, F-14000 Caen, France

${ }^{2}$ CHU de Caen, Service ORL et Chirurgie cervico-faciale, F-14000 Caen, France

\author{
* Auteur correspondant. \\ e-mail : lisemarie61@ yahoo.fr (L.-M. Roussel) \\ Hôpital Côte de Nacre, Avenue Côte de Nacre, 14000 Caen0231063106
}

\section{Remerciements}

Nous remercions les patients et leurs conjoints qui ont rempli et renvoyé nos questionnaires, les ORL, les chirurgiens maxillo-faciaux, les stomatologues, les dentistes, les oncologues, les anatomo-pathologistes, les médecins d'assurance maladie, les secrétaires de réunions multidisciplinaires, les secrétaires médicales et les archivistes du Calvados, de la Manche, de la Somme et du Nord. Nous remercions également la Ligue Nationale Contre le Cancer et les comités départementaux de la Ligue Contre le Cancer du Calvados, de la Manche et du Nord pour le financement du projet (grant number : PRC07 / EB).

\section{Conflits d'intérêt : Aucun}




\section{Résumé}

On définit la sociabilité comme étant l'ensemble des expériences reliant l'individu à d'autres personnes. Il s'agit de la première étude s'intéressant spécifiquement à l'impact du cancer ORL sur la sociabilité des conjoints.

Le recueil a été réalisé via un questionnaire original envoyé aux conjoints des patients. Le critère de jugement principal retenu a été l'auto-évaluation par le conjoint de l'influence ou non de la maladie sur sa vie quotidienne. L'impact de la sociabilité sous ces différents aspects a également été étudié : l'environnement amical, l'environnement inconnu, l'environnement extérieur connu, l'environnement nécessaire et les activités solitaires.

Deux cent soixante-dix conjoints ont répondu au questionnaire. Leurs activités quotidiennes étaient influencées par la maladie dans $71,5 \%$ des cas.

L'environnement amical a été jugé détérioré dans 46,4\%. L'environnement inconnu a été jugé détérioré dans $44,0 \%$ des cas. Les pratiques sociales liées à l'environnement extérieur connu ont diminué chez $67,8 \%$ des conjoints. L'environnement nécessaire a été jugé altéré chez $26,0 \%$ des conjoints. Le nombre d'activités solitaires a augmenté dans 35,6\% des cas.

L'impact social pour les conjoints des patients est donc majeur.

\section{Mots clefs}

Cancer ORL, qualité de la vie, conjoint, sociabilité 


\section{Introduction}

On définit la sociologie comme l'analyse de l'organisation sociale et de l'étude de l'homme en société[1]. Son champ d'étude est donc très vaste, allant du domaine de l'économie, au domaine politique ou encore aux sciences humaines. Elle s'intéresse également à l'organisation dans le domaine de la santé[2,3] et aux conséquences de la maladie sur la qualité de vie des patients[4] et de leur entourage[5].

Cette altération de la qualité de vie[6] est particulièrement importante en ORL en raison des troubles de la communication[7], de la déglutition[8] et des séquelles esthétiques[9] qui en résultent.

Pourtant aucune étude ne s'est intéressée spécifiquement aux retentissements en termes de sociabilité, qui est un autre champ d'étude en sociologie. On définit la sociabilité comme étant l'ensemble des expériences reliant l'individu à d'autres personnes[10]. La sociabilité regroupe l'analyse de cinq environnements différents: l'environnement amical, l'environnement solitaire, l'environnement connu, l'environnement inconnu, et l'environnement nécessaire[10].

Les questionnaires les plus communément utilisés en ORL, à savoir EORTC QLQC30 et son module H\&N35[11] dédié à l'ORL, n'aborde que partiellement l'impact de la maladie sur la sociabilité: 12 questions sur 65 s'y intéressent (annexes 1 et 2). L'environnement solitaire n'est pas évalué et les autres environnements ne le sont que partiellement. De plus, ces questionnaires ne sont applicables qu'au patient et pas à leur conjoint.

On note qu'il n'existe pas de questionnaire spécifique validé évaluant la sociabilité des individus.

L'objectif de cette étude est d'évaluer l'impact du cancer ORL sur la sociabilité des conjoints en utilisant un questionnaire original.

\section{Matériel et Méthode}

\section{- Population}

Nous avons inclus les conjoints de patient ayant présenté un carcinome épidermoïde du larynx, de l'hypopharynx, de l'oropharynx, et de la cavité buccale entre le 1er janvier 2008 et le 31 décembre 2010 de quatre départements français (Calvados, Manche, Nord, et Somme) à partir des registres des tumeurs. 
Les conjoints de patients présentant une autre localisation tumorale, une autre maladie grave, une seconde localisation ORL ou une récidive tumorale ont été exclus.

Les conjoints des patients sélectionnés ont reçu un questionnaire par la poste évaluant leurs relations sociales 1 mois après le début de la prise en charge thérapeutique (annexe 3). Seuls les questionnaires répondus dans les 12 mois après le début de la prise en charge thérapeutique ont été inclus. Un consentement éclairé des patients et de leurs conjoints a été dans le même temps recueilli.

Les caractéristiques de la maladie (stade tumoral, localisation et le type de traitement) ont été obtenues grâce aux données des registres des tumeurs. Les stades tumoraux TNM ont été répartis en deux groupes : stade précoce (stade I et II) et stade avancé (stade III et IV). Les localisations tumorales ont été regroupées en localisation supra et infra-hyoïdienne (tableau 1). Le type de prise en charge thérapeutique a également été défini comme suit : chirurgie, radiothérapie, ou chimiothérapie exclusive ou soins de conforts. Les cas où les séquelles esthétiques du malade ont été jugées importantes par le conjoint ont été recueillis.

Les caractéristiques des conjoints ont également été recueillies (âge, sexe, activité professionnelle, revenus) (tableau 1). Ces données ont été incluses dans les questionnaires adressés aux conjoints.

Le protocole d'étude a été approuvé par le Conseil d'éthique des établissements.

\section{- Critères de jugement}

Le critère de jugement principal retenu a été l'auto-évaluation par le conjoint de l'influence ou non de la maladie sur sa vie quotidienne.

Pour évaluer les différents niveaux de sociabilité, cinq environnements sociaux ont été définis de sorte que les questions soient regroupées de la façon suivante :

- Les questions s'intéressant à l'environnement amical : incluent les relations amicales, familiales et de l'entourage proche.

- Les questions s'intéressant à l'environnement inconnu : incluent les sorties dans des lieux inconnus avec des personnes inconnues.

- Les questions s'intéressant à l'environnement connu : incluent les sorties dans des lieux connus mais en présence de personnes inconnues.

- Les questions s'intéressant à l'environnement nécessaire : incluent les rapports au sein même du couple. 
- Les questions s'intéressant à l'environnement solitaire : activités réalisées par le conjoint tout seul incluant son activité professionnelle.

Le niveau d'information ressenti par le conjoint vis-à-vis de l'état de santé du patient a aussi été évalué ainsi que le retentissement somatique (troubles du sommeil).

\section{- Statistiques}

Le critère de jugement principal évaluant l'influence ou non de la maladie sur la vie quotidienne du conjoint a été exprimé en pourcentage. Le questionnaire de satisfaction sur l'information reçue a elle aussi été exprimée en pourcentage (pourcentage de «satisfaits» versus pourcentage de «non satisfaits »). Le retentissement somatique (trouble du sommeil) a été exprimé en pourcentage.

Pour les autres questions, un score de -1 à +1 a été donné aux réponses indiquant respectivement une diminution ou une augmentation de la fréquence ou de la qualité des activités étudiées.

Une augmentation de la fréquence des activités étudiées dénote une amélioration de la sociabilité alors qu'une diminution de la fréquence des activités étudiées dénote une détérioration de la sociabilité sauf pour les activités solitaires (lecture et télévision). Dans ce dernier cas une augmentation de la fréquence des activités solitaires dénote une détérioration de la sociabilité et a été cotée -1 alors qu'une diminution de leur fréquence était cotée +1 .

Un score de 0 a été donné aux réponses n'indiquant aucun changement («comme avant», « identiques », « aucun changement »).

En l'absence de réponse à la question, le score de 0 était imputé. Nous avons considéré que dans ce dernier cas, le conjoint ne s'identifiait pas aux pratiques étudiées et n'avait probablement pas changé son comportement.

Pour chaque environnement la détérioration a été exprimée en pourcentage.

Enfin une analyse univariée a été effectuée par régression logistique avec un niveau de signification de 5\%. Celle-ci a permis de tester l'influence des différents facteurs (revenus, type de traitement, sexe, âge, activité professionnelle, localisation tumorale, présence de troubles du sommeil chez le conjoint et présence de séquelles esthétiques jugées importantes) 
sur la probabilité d'avoir une dégradation des environnements définis ci-dessus. Les analyses ont été effectuées avec SAS ® 9.3.

\section{Résultats}

\section{- Population}

Nous avons envoyé le questionnaire aux conjoints des 1525 patients présentant les critères d'éligibilité. Deux cent soixante-dix conjoints ont répondu au questionnaire et ont ainsi été inclus (figure 1).

Parmi les conjoints, il y avait 236 femmes et 34 hommes âgés de moins de 60 ans dans 61,1\% des cas. Dans la majorité des cas ces derniers occupaient une activité professionnelle au moment du recueil (51,9\% des cas) et les revenus cumulés du couple étaient supérieurs à 1099 euros dans $64,1 \%$ des cas (tableau 1).

La maladie était à un stade avancé dans $64,1 \%$ des cas et avait nécessité un traitement chirurgical dans $41,9 \%$ des cas (tableau 1). Les séquelles esthétiques chez le malade ont été jugées importantes par le conjoint dans $41,9 \%$ des cas.

\section{- Critère de jugement principal}

Les conjoints ont estimé que leurs activités quotidiennes étaient influencées par la maladie dans $71,5 \%$ des cas.

\section{- Environnement amical}

L'environnement amical a été jugé détérioré chez 46,4\% des conjoints.

Il était significativement détérioré lorsque le stade de la tumeur était à un stade avancé $(\mathrm{OR}=$ $1,88[1,13-3,13], \mathrm{p}<0.05)$, que le patient présentait des séquelles esthétiques jugées importantes par le conjoint $(\mathrm{OR}=1,75[1,07-2,85]$, $\mathrm{p}<0.05)$ ou qu'il avait été traité par radiothérapie $(\mathrm{OR}=2.46[1.31-4,60] \mathrm{p}<0,01)$.

Si les revenus du couple étaient de plus de 1099 euros / mois, l'environnement amical était significativement détérioré $(\mathrm{OR}=2,34[1,26-4,35] \mathrm{p}<0,01)$. Il en était de même lorsque le conjoint était un homme ( $\mathrm{p}<0,05)$. 
Des troubles du sommeil chez le conjoint s'accompagnaient d'une altération significative de cet environnement $(\mathrm{OR}=3.26[1.82-5.82], p<.001)$.

Les autres facteurs ne sont pas associés à l'altération de cet environnement.

\section{- Environnement inconnu}

Près d'un conjoint sur deux $(44,0 \%)$ avait une altération de l'environnement inconnu.

Les conjoints des patients traités par radiothérapie avaient une altération significative de l'environnement inconnu (OR = 2,02 [1,09-3,75], $\mathrm{p}<0,05)$.

Des troubles du sommeil chez le conjoint s'accompagnaient d'une altération significative de cet environnement $(\mathrm{OR}=2,41[1,36-4,25], p<0,01)$.

Les autres facteurs n'étaient pas associés à l'altération de cet environnement.

\section{- Environnement connu}

Les pratiques sociales liées à l'environnement extérieur connu ont diminué chez 67,8\% des conjoints.

Cet environnement était significativement altéré lorsque le patient était traité par radiothérapie $(\mathrm{OR}=2,06[1,13-3,74], \mathrm{p}<0,05)$.

Des troubles du sommeil chez le conjoint s'accompagnait d'une altération significative de cet environnement $(\mathrm{OR}=1.95[1,11-3,43], p<0,05)$.

Les autres facteurs n'étaient pas associés à l'altération de cet environnement.

\section{- Environnement nécessaire}

L'environnement nécessaire a été jugé altéré chez 26,0\% des conjoints.

Cette altération était significative lorsque les patients étaient traités par radiothérapie $(\mathrm{OR}=$ $2,20[1,02-4,75], \mathrm{p}<0,05)$.

Des troubles du sommeil chez les conjoints s'accompagnait d'une altération significative de cet environnement $(\mathrm{OR}=2.12[1,08-4,16], p<0,05)$.

Les autres facteurs n'étaient pas associés à l'altération de cet environnement. 


\section{- Environnement solitaire}

Le nombre d'activités solitaires est resté inchangé chez $64,4 \%$ des conjoints et a augmenté dans $35,6 \%$ des cas.

Les conjoints des patients ayant subi des séquelles esthétiques jugées importantes $(\mathrm{OR}=1,68$ [1,01-2,78], $\mathrm{p}<0,05)$ ont augmenté significativement leur activité solitaire.

Les couples ayant un revenu supérieur ou égal au salaire minimum $(\mathrm{OR}=1,99$ [1,03-3,83], $\mathrm{p}$ $<0,05)$ ou âgés de 60 ans ou plus au moment du diagnostic $(\mathrm{OR}=2,36[1,37-4,07], \mathrm{p}<0,01)$ ont considérablement augmenté leur activité solitaire.

Les autres facteurs n'étaient pas associés à l'altération de cet environnement.

\section{- Information et retentissement somatique}

Plus des trois quarts des partenaires $(77,1 \%)$ semblaient avoir été bien informés sur la maladie. Les troubles du sommeil étaient quant à eux présents chez $68,1 \%$ des conjoints.

\section{Discussion}

Les conjoints ont estimé que leurs activités quotidiennes étaient influencées par la maladie dans $71,5 \%$ des cas. Tous les aspects de la sociabilité étaient affectés par la maladie du conjoint dans au moins un quart des cas.

La qualité de vie des conjoints de patients souffrant d'un cancer de la tête et du cou est connue pour être détériorée après l'annonce du diagnostic[12-15]. Cependant, les questionnaires utilisés n'incluent pas la sociabilité[15]. Notre étude est la première à montrer l'impact de la maladie sur la sociabilité des conjoints.

L'environnement qui semble le moins affecté par la maladie est regroupé dans les activités solitaires avec une augmentation des activités solitaires dans un quart des cas. Nous avons considéré l'augmentation de ce type d'activités comme étant une détérioration en termes de sociabilité bien que ce dernier point soit discutable. En effet, il peut être considéré comme un temps personnel nécessaire pour certains. Par ailleurs nous avons exclu de cet environnement la fréquence des appels téléphoniques qui semble plutôt protéger les conjoints de l'isolement social. On note par ailleurs que les conjoints de patient ayant des séquelles esthétiques 
importantes ont significativement augmenté leurs activités solitaires, essentiellement chez les couples aux revenus les plus aisés. Ces résultats semblent en accord avec les précédents travaux sur le sujet[16,17].

L'analyse univariée qui a été proposée dans ce travail peut toutefois être discutée. Il existe très probablement des facteurs confondants et une analyse multivariée aurait été préférable. Toutefois le nombre important de données manquantes n'a pas permis de réaliser ce type d'analyse.

La radiothérapie semble significativement associée à une altération de l'environnement amical, inconnu, connu et nécessaire. Là encore il peut s'agir d'un facteur confondant dans la mesure où les patients ayant reçu de la radiothérapie sont aussi ceux ayant un stade tumoral avancé. Or les stades tumoraux les plus avancés sont aussi ceux ayant le plus de séquelles esthétiques[18], de troubles de la déglutition[8,19], d'altération de l'état général[20] ou encore de modification de la voix[21]. Une analyse multivariée aurait encore été dans ce cas intéressante.

L'environnement amical était significativement détérioré lorsque le conjoint était un homme. En France, l'âge moyen des patients atteints de cancer de la tête et du cou est de 55 ans[22] et, selon l'Institut national de la statistique et de l'économie (INSEE), les hommes de ce groupe d'âge dépendent davantage de leurs partenaires pour les tâches ménagères et dans les activités de la vie quotidienne[23]. Avoir des amis à la maison devient difficile lorsque son conjoint a un cancer de la tête et du cou.

Nous avons utilisé comme critère de jugement principal une question fermée ne permettant qu'une réponse qualitative binomiale. Pour préciser l'impact sur la sociabilité des conjoints, un questionnaire permettant une réponse quantitative aurait pu être préféré.

La présence de séquelles esthétiques jugées importantes renforce l'isolement et altère la sociabilité. Goffman utilise le terme de «stigmate »[24] pour désigner un attribut indésirable qui rend un individu différent de la norme ou du groupe social dans lequel il vit. La stigmatisation de ces patients est liée à un attribut ou séquelle physique comme la perte de voix qui jette le discrédit sur l'individu et peut constituer une barrière à l'interaction sociale. Les relations avec les autres deviennent difficiles, surtout quand les séquelles sont visibles. Les patients souffrant de cette stigmatisation peuvent se sentir aliénés par les gens qu'ils rencontrent. Les gens «normaux » ne savent pas très bien comment réagir à la stigmatisation et un sentiment d'inconfort ou d'évitement se produit.

Enfin, nous nous sommes intéressés uniquement aux conjoints des patients à un stade précoce de leur prise en charge : entre le 1er et le 12e mois après le diagnostic. Les effets secondaires 
tardifs des traitements ne sont pas tous encore survenus. La radiothérapie, en particulier, peut entraîner des effets secondaires à plus long terme[25]. Une étude à plus long terme de l'impact de la maladie sur les conjoints aurait pu être réalisée.

\section{Conclusion}

Bien que de nombreuses études aient montré l'impact du cancer sur la qualité de vie des patients et des conjoints, notre étude est la première montrant la détérioration de la sociabilité des conjoints. Il ne faut pas négliger le besoin de soutien des conjoints. Les thérapeutes doivent être conscients de ces changements et une gestion multidisciplinaire devrait être entreprise pour mieux les soutenir. 
Références

[1] Gay T. L'indispensable de la sociologie. Studyrama; 2006.

[2] Babin E, Grandazzi G. Cooperation between medicine and sociology in head and neck oncology. Otolaryngol--Head Neck Surg Off J Am Acad Otolaryngol-Head Neck Surg 2014;150:712-5. doi:10.1177/0194599814521783.

[3] Carricaburu D, Ménoret M. Sociologie de la santé: Institutions, professions, maladies. Armand Colin; 2004.

[4] Babin E, Sigston E, Hitier M, Dehesdin D, Marie JP, Choussy O. Quality of life in head and neck cancers patients: predictive factors, functional and psychosocial outcome. Eur Arch Oto-Rhino-Laryngol Off J Eur Fed Oto-Rhino-Laryngol Soc EUFOS Affil Ger Soc OtoRhino-Laryngol - Head Neck Surg 2008;265:265-70. doi:10.1007/s00405-007-0561-0.

[5] Richardson AE, Morton RP, Broadbent EA. Changes over time in head and neck cancer patients' and caregivers' illness perceptions and relationships with quality of life. Psychol Health 2016;31:1203-19. doi:10.1080/08870446.2016.1203686.

[6] Babin E, Heutte N, Grandazzi G, Prévost V, Robard L. [Quality of life and supportive care in head and neck cancers]. Bull Cancer (Paris) 2014;101:505-10. doi:10.1684/bdc.2014.1960.

[7] Arenaz Búa B, Pendleton H, Westin U, Rydell R. Voice and swallowing after total laryngectomy. Acta Otolaryngol (Stockh) 2017:1-5. doi:10.1080/00016489.2017.1384056.

[8] van den Berg MGA, Rütten H, Rasmussen-Conrad EL, Knuijt S, Takes RP, van Herpen CML, et al. Nutritional status, food intake, and dysphagia in long-term survivors with head and neck cancer treated with chemoradiotherapy: a cross-sectional study. Head Neck 2014;36:60-5. doi:10.1002/hed.23265.

[9] Fingeret MC, Teo I, Goettsch K. Body image: a critical psychosocial issue for patients with head and neck cancer. Curr Oncol Rep 2015;17:422. doi:10.1007/s11912-014-0422-0.

[10] Bidart C. Sociabilités : quelques variables. Rev Fr Sociol 1988;29:621-48. doi:10.2307/3321515.

[11] Bjordal K, de Graeff A, Fayers PM, Hammerlid E, van Pottelsberghe C, Curran D, et al. A 12 country field study of the EORTC QLQ-C30 (version 3.0) and the head and neck cancer specific module (EORTC QLQ-H\&N35) in head and neck patients. EORTC Quality of Life Group. Eur J Cancer Oxf Engl 1990 2000;36:1796-807.

[12] Katz MR, Irish JC, Devins GM, Rodin GM, Gullane PJ. Psychosocial adjustment in head and neck cancer: the impact of disfigurement, gender and social support. Head Neck 2003;25:103-12. doi:10.1002/hed.10174.

[13] Mathieson CM, Logan-Smith LL, Phillips J, MacPhee M, Attia EL. Caring for head and neck oncology patients. Does social support lead to better quality of life? Can Fam Physician Med Fam Can 1996;42:1712-20.

[14] Morton RP. Studies in the quality of life of head and neck cancer patients: results of a two-year longitudinal study and a comparative cross-sectional cross-cultural survey. The Laryngoscope 2003;113:1091-103. doi:10.1097/00005537-200307000-00001.

[15] Vickery LE, Latchford G, Hewison J, Bellew M, Feber T. The impact of head and neck cancer and facial disfigurement on the quality of life of patients and their partners. Head Neck 2003;25:289-96. doi:10.1002/hed.10206.

[16] Smith JD, Shuman AG, Riba MB. Psychosocial Issues in Patients with Head and Neck Cancer: an Updated Review with a Focus on Clinical Interventions. Curr Psychiatry Rep 2017;19:56. doi:10.1007/s11920-017-0811-9.

[17] Suzuki M, Deno M, Myers M, Asakage T, Takahashi K, Saito K, et al. Anxiety and depression in patients after surgery for head and neck cancer in Japan. Palliat Support Care 
2016;14:269-77. doi:10.1017/S1478951515000930.

[18] Yeh DH, Sahovaler A, Fung K. Reconstruction after salvage laryngectomy. Oral Oncol 2017;75:22-7. doi:10.1016/j.oraloncology.2017.10.009.

[19] Hedström J, Tuomi L, Finizia C, Olsson C. Correlations Between Patient-Reported Dysphagia Screening and Penetration-Aspiration Scores in Head and Neck Cancer Patients Post-oncological Treatment. Dysphagia 2017. doi:10.1007/s00455-017-9847-6.

[20] Creighton F, Lin A, Leavitt E, Lin D, Deschler D, Emerick K. Factors affecting survival and locoregional control in head and neck cSCCA with nodal metastasis. The Laryngoscope 2017. doi:10.1002/lary.27048.

[21] van Sluis KE, van der Molen L, van Son RJJH, Hilgers FJM, Bhairosing PA, van den Brekel MWM. Objective and subjective voice outcomes after total laryngectomy: a systematic review. Eur Arch Oto-Rhino-Laryngol Off J Eur Fed Oto-Rhino-Laryngol Soc EUFOS Affil Ger Soc Oto-Rhino-Laryngol - Head Neck Surg 2018;275:11-26. doi:10.1007/s00405-017-4790-6.

[22] Brugère J. Les cancers des voies aérodigestives supérieurs 2002.

[23] INSEE. Depuis 11 ans, moins de tâches ménagères, plus d'Internet 2011.

[24] Goffman E. Stigma: Notes on the Management of Spoiled Identity. Simon and Schuster; 2009.

[25] Bibault J-E, Dussart S, Pommier P, Morelle M, Huguet M, Boisselier P, et al. Clinical Outcomes of Several IMRT Techniques for Patients With Head and Neck Cancer: A Propensity Score-Weighted Analysis. Int J Radiat Oncol Biol Phys 2017;99:929-37. doi:10.1016/j.ijrobp.2017.06.2456. 


\begin{tabular}{|c|c|c|}
\hline Variables & $\mathbf{N}$ (nombre) & $\begin{array}{c}\% \\
\text { (pourcentage) }\end{array}$ \\
\hline \multicolumn{3}{|l|}{ Caractéristiques de la maladie } \\
\hline \multicolumn{3}{|l|}{ Localisation } \\
\hline Supra-hyoïdienne & 156 & 57,8 \\
\hline Sous-hyoïdienne & 114 & 42,2 \\
\hline \multicolumn{3}{|l|}{ Stade } \\
\hline I-II & 97 & 35,9 \\
\hline III-IV & 173 & 64,1 \\
\hline \multicolumn{3}{|l|}{ Traitement } \\
\hline Chirurgie & 113 & 41,9 \\
\hline Radiothérapie initiale ou adjuvante & 212 & 78,5 \\
\hline $\begin{array}{l}\text { Soins de confort ou chimiothérapie } \\
\text { exclusive }\end{array}$ & 58 & 21,5 \\
\hline \multicolumn{3}{|l|}{ Caractéristiques du conjoint } \\
\hline \multicolumn{3}{|l|}{ Age } \\
\hline$<60$ ans & 165 & 61,1 \\
\hline$\geq 60$ ans & 103 & 38,2 \\
\hline Données manquantes & 2 & 0,7 \\
\hline \multicolumn{3}{|l|}{ Sexe } \\
\hline Homme & 34 & 12,6 \\
\hline Femme & 236 & 87,4 \\
\hline \multicolumn{3}{|l|}{ Activité professionnelle } \\
\hline Sans emploi & 127 & 47,0 \\
\hline Avec activité professionnelle & 140 & 51,9 \\
\hline Données manquantes & 3 & 1,1 \\
\hline \multicolumn{3}{|c|}{ Salaire cumulé mensuel du conjoint et du patient } \\
\hline$\leq 1099$ euros & 61 & 22,6 \\
\hline$>1099$ euros & 173 & 64,1 \\
\hline Données manquantes & 36 & 13,3 \\
\hline
\end{tabular}

Tableau 1: Caractéristiques des patients et de leurs conjoints. 
Figure 1. Population étudiée

Annexe 1. Questionnaire EORTC QLQ-C30 avec les questions s'intéressant à la sociabilité encadrées en rouge Annexe 2. Questionnaire EORTC QLQ-H\&N35 avec les questions s'intéressant à la sociabilité encadrées en rouge

Annexe 3. Questionnaire originale utilisé dans notre étude et destiné aux conjoints des patients, 1 mois après le début de la prise en charge thérapeutique 
Population éligible

$\mathbf{N}=1525$

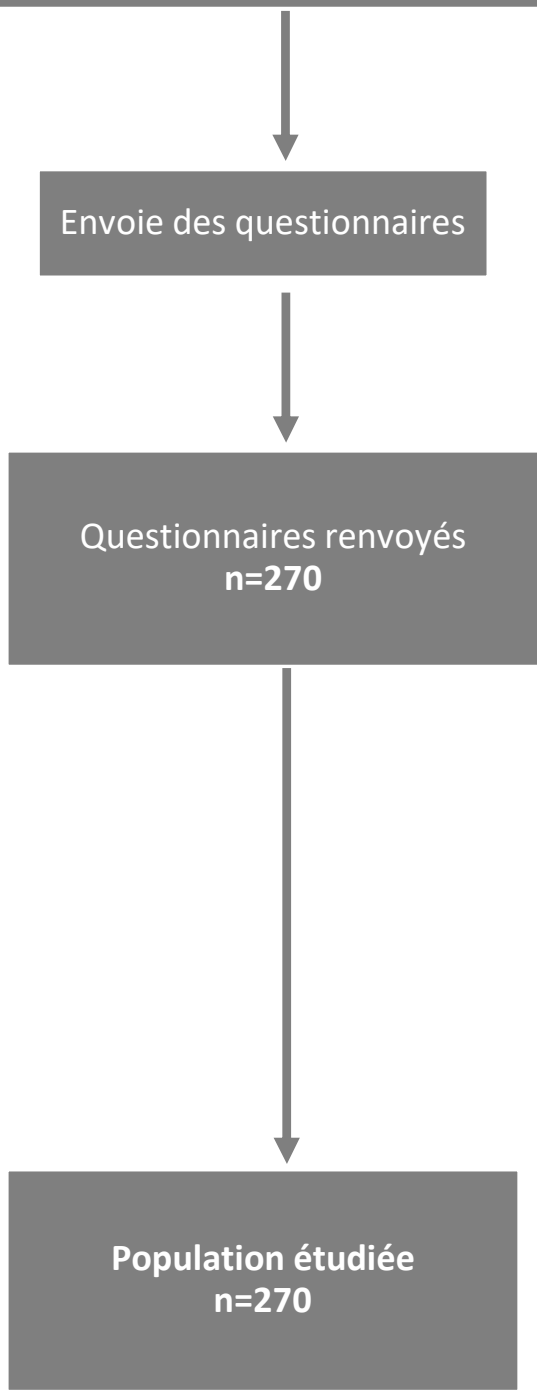

Figure 1. Population étudiée 